UDK 528.4

\title{
BODENORDNUNG IN STÄDTISCHEN VERDICHTUNGSGEBIETEN DER BUNDES REPUBLIK DEUTSCHLAND
}

\author{
Erich Weiß \\ Institut für Städtebau, Bodenordnung und Kulturtechnik Rheinische Friedrich-Wilhelms Universität Bonn Meckenheimer, \\ Allee 172, D-53115 Bonn, E-Post: probobo@uni-bonn.de
}

Eingang der Manuskripts: 2001 2003; angenommen 24032004

\begin{abstract}
Zusammenfassung: Die wesentlichen Aspekte der besonderen Bedeutung des Grundeigentums in der Bundesrepublik Deutschland werden zusammengestellt. Daraus werden die Aufgaben der öffentlich-rechtlichen Bodenordnung in der Bundesrepublik Deutschland abgeleitet und das zugrundeliegende System der Bodennutzungsplanung erläutert. Schlußendlich werden für die Bundesrepublik Deutschland die wichtigen öffentlich-rechtlichen Bodenordnungsinstrumente mit ihren privatnützigen und fremdnützigen Ausprägungen vorgestellt.
\end{abstract}

Deskriptoren: Bodeneigentum, Bodenordmung, Bodennutzplanung

\section{Zur Bedeutung des Bodeneigentums}

Die Rechtsordnung der Bundesrepublik Deutschland, eines freiheitlichen, demokratischen und sozialen Rechtsstaates, wird entscheidend geprägt durch das Verhältnis der allgemeinen Freiheitsrechte nach Artikel 2 Grundgesetz (GG) und der Eigentumsgewährleistung nach Artikel 14 Grundgesetz ${ }^{1}$.

In Artikel 2 GG heißt es:

"Artikel 2 GG - Allgemeines Freiheitsrecht

(1) Jeder hat das Recht auf die freie Entfaltung seiner Persönlichkeit, soweit er nicht die Rechte anderer verletzt und nicht gegen die verfassungsmäßige Ordnung oder das Sittengesetz verstößt.

(2) Jeder hat das Recht auf Leben und körperliche Unversehrtheit. Die Freiheit der Person ist unverletzlich. In diese Rechte darf nur auf Grund eines Gesetzes eingegriffen werden" ${ }^{2)}$

In Artikel 14 Grundgesetz heißt es:

"Artikel $14 \mathrm{GG}$ - Eigentum, Erbrecht und Enteignung

(1) Das Eigentum und das Erbrecht werden gewährleistet. Inhalt und Schranken werden durch die Gesetze bestimmt.

(2) Eigentum verpflichtet. Sein Gebrauch soll zugleich dem Wohle der Allgemeinheit dienen.

(3) Die Enteignung ist nur zum Wohle der Allgemeinheit zulässig. Sie darf nur durch Gesetz oder auf Grund eines Gesetzes erfolgen, das Art und Ausmaß der ${ }^{1}$ Entschädigung regelt. Die Entschädigung ist unter gerechter Abwägung der Interessen der Allgemeinheit und der Beteiligten $\mathrm{zu}$ bestimmen. Wegen der Höhe der

\footnotetext{
${ }^{1)}$ Das Grundgesetz der Bundesrepublik Deutschland enthält die Rechtsgrundlagen mit Verfassungsrang.

${ }^{2)}$ Art. 2 GG enthält also insgesamt folgende vier Grundrechte: Abs. 1 Satz 1 - das Recht auf freie Entfaltung der Persönlichkeit; Absatz 2 Satz 1 - das Recht auf Leben; das Recht auf körperliche Unversehrtheit; Abs. 2 Satz 2 - das Recht auf Freiheit der Person.
}

Entschädigung steht im Streitfalle der Rechtsweg vor den ordentlichen Gerichten offen." ${ }^{3)}$

Bereits der große Staatsmann und Vordenker der sogenannten abendländischen Kultur, Aristoteles, hat dreihundert Jahre vor unserer Zeitrechnung diesen untrennbaren Zusammenhang zwischen Freiheit und Eigentum klar erkannt und formuliert, daß die Entwicklung zu einer freiheitlichen Demokratie an die Existenz von Menschen gebunden ist, die über Bildung und Eigentum verfügen (Berlin und Stuttgart 1855-1900).

In der Geschichte haben wiederholt große Enteignungs- bzw. Konfiskationswellen unser Land überflutet $^{4)}$. Dabei sei nur an folgendes erinnert:

-Im Jahre 1789 ließ Robespierre während der Französischen Revolution Menschen enthaupten und ihren Besitz einziehen, um eine bessere Gesellschaftsordnung zu errichten.

-Im Jahre 1803 entzog Napoleon I während der Säkularisation den Schlössern, Klöstern und Kirchen ihren Besitz, um die Wurzeln des Feudalismus zu beseitigen und die Prinzipien von Freiheit und Gleichheit für alle Menschen durchzusetzen.

-In den Jahren ab 1933 entwürdigten die Hitlerschergen unsere jüdischen Mitbürger und raubten ihnen das Eigentum als Folge der herrschenden antisemitischen Ideologie. Deshalb manifestiert das Gesetz zur Wiedergutmachung nationalsozialistischen Unrechts,

\footnotetext{
${ }^{3)}$ Artikel 14 GG enthält also in seiner Systematik folgendes: Abs. 1 Satz 1 - das Grundrecht und das Rechtsinstitut; Abs. 1 Satz 2 - den Regelungsauftrag zur Inhalts- und Schrankenbestimmung; Abs. 2 - die Sozialpflichtigkeit; Abs. 3 - die Enteignung mit der Entschädigung.

4) Enteignung ist danach eine rechtmäßige, vermögensmindernde Beeinträchtigung durch hoheitlichen Eingriff, die den Betroffenen gegenüber anderen ungleich oder unzumutbar schwer belastet. Konfiskation ist dabei ein entschädigungsloser Eigentumsentzug, der nicht primär zur Güterbeschaffung für die öffentliche Hand oder von dieser im öffentlichen Interesse geförderter Privater erfolgt, sondern als personenbezogener Eingriff oder als eigentumsrechtliche Strafmaßnahme gegenüber einer bestimmten Personengruppe.
} 
welches auf Absprachen zwischen Konrad Adenauer und David Ben Gurion beruht, die Rückkehr der Bundesrepublik Deutschland in die geistesgeschichtliche Welt von Freiheit und Eigentum des westlichen Europas.

-In den Jahren ab 1945 entzog die sowjetische Besatzungsmacht in den fünf östlichen Provinzen Deutschlands zunächst mittels sogenannter Bodenreform, dann durch die Kollektivierung der Landwirtschaft der Bevölkerung in großem Umfange das Grundeigentum und schließlich durch den Mauerbau von 1961 die Freiheit, um die eigenen Machtverhältnisse abzusichern [1].

Der russische Soziologe Fedor Stepun, der in den 20er Jahren in Dresden lehrte, hat bei diesen Zusammenhängen einmal darauf hingewiesen, daß Rußland infolge seines byzantinischen Christentums, nicht wie Rom, vom aristotelischen Naturrecht nicht geprägt sei und daher den europäischen Eigentumsbegriff nicht übernommen habe; deshalb fehle seinen Landsleuten möglicherweise eine Vorstellung von Freiheit und Unabhängigkeit, wie sie sich im westlichen Europa entwickelt habe. Vielleicht sei es gerade deshalb Lenin im Jahre 1917 gelungen, entgegen aller marxistisch-revolutionären Theorien in Rußland die Macht zu ergreifen und mittels Enteignung bzw. Konfiskation seine Gesellschaftsordnung zu errichten. Konsequent erscheint daher auch die Politik der sowjetischen Besatzungsmacht nach dem zweiten Weltkrieg in Deutschland oder andererseits das Scheitern des kommunistischen Systems gerade in Polen sowie in aller Stringenz der verfassungsmäßige Verzicht der Irischen Republik auf eine Enteignung [2].

Der Bundesgerichtshof als oberstes Zivilgericht der Bundesrepublik Deutschland hat schließlich zu dem hier nur kurz angerissenen Gesamtsachverhalt bereits in seiner Grundsatzentscheidung vom 10. Juni 1952 geäußert: "Der in den Staat eingegliederte Einzelne bedarf, um unter seinesgleichen als Person, d. h. frei und selbstverantwortlich leben zu können, und um nicht zum bloßen Objekt einer übermächtigen Staatsgewalt zu werden, also um seiner Freiheit und Würde willen, einer rechtlich streng gesicherten Sphäre des Eigentums."

Das Bundesverfassungsgericht als höchstes Gericht der Bundesrepublik Deutschland hat im Urteil vom 18. Dezember 1968 ergänzend bestätigt: "Das Eigentum ist ein elementares Grundrecht, das in einem inneren Zusammenhang mit der Garantie der persönlichen Freiheit steht. Ihm kommt im Gesamtgefüge der Grundrechte die Aufgabe zu, dem Träger des Grundrechts einen Freiheitsraum im vermögensrechtlichen Bereich sicherzustellen und ihm damit eine eigenverantwortliche Gestaltung des Lebens zu ermöglichen. Die Garantie des Eigentums als Rechtseinrichtung dient der Sicherung dieses Grundrechts."

Diese Gedankengänge verdeutlichen die grundlegende Bedeutung von Eigentum und insbesondere von Grundeigentum, weil dieses Gut nicht beliebig vermehrbar ist. In diesem Zusammenhang stellt sich sogleich auch die Frage, inwieweit die Eigentumssicherung privatisiert werden kann, werden doch elementare Grundrechte des Menschen davon betroffen.

Ich denke, dieses beleuchtet zugleich auch die Schwierigkeiten, die sich bei der Annäherung so grund- verschiedener Rechtssysteme aus Osteuropa und Westeuropa heute noch zeigen. Mögen die politisch Verantwortlichen hier nur die Orientierung in diesen Wertestrukturen nicht verlieren.

\section{Zum Begriff der Bodenordnung}

Die Bodenordnung in der Bundesrepublik Deutschland umfaßt eine statische und eine dynamische Komponente. Die statische Komponente der Bodenordnung beinhaltet die Eigentums- (Rechts-)Verfassung des Grund und Bodens einschließlich seiner Nutzung und Besteuerung. Die dynamische Komponente der Bodenordnung beinhaltet alle Maßnahmen, die dazu dienen, die Eigentums-, Besitz- und Nutzungsverhältnisse am Grund und Boden - die subjektiven Rechtsverhältnisse - möglichst weitgehend mit den in der Bodennutzungsplanung dokumentierten Ansprüchen an dessen Nutzung die objektiven Planungsziele - in Übereinstimmung zu bringen und störende externe Effekte in der planungskonformen Nutzung zu eliminieren, also private und öffentliche Interessengegensätze aufzulösen. Dabei werden als Bodennutzungsplanung die Teile der Raumordnung, Landesplanung, Fachplanung und Bauleitplanung zusammengefaßt, die unmittelbar für die Nutzung des Grund und Bodens und nicht für die darauf stehenden Bauwerke und deren Gestaltung gelten.

Die Bodenordnungsmaßnahmen können, je nachdem, ob sie vorrangig aus der land- und forstwirtschaftlichen oder der städtebaulichen Planungsmotivation resultieren, in ländliche oder städtebauliche Bodenordnungen unterschieden werden.

Die Bodenordnungsmaßnahmen können aber auch nach ihren rechtlichen Grundlagen in privatrechtliche oder öffentlich-rechtliche bzw. in freiwillig oder von Amts wegen durchgeführte Bodenordnungen unterschieden werden.

Die privatrechtlichen Bodenordnungsmaßnahmen in der Bundesrepublik Deutschland sind nach dem Bürgerlichen Gesetzbuch (BGB) zu unterscheiden in Kauf, Tausch, Schenkung und Teilung. Hierauf wird nachfolgend nicht mehr näher eingegangen.

Die öffentlich-rechtlichen Bodenordnungsmaßnahmen in der Bundesrepublik Deutschland (im engeren Sinne) können nach der Gesetzsystematik weiter unterschieden werden in die Umlegung und die Grenzregelung nach dem Baugesetzbuch $(\mathrm{BauGB})^{5}$, sowie in die Flurbereinigung, die vereinfachte Flurbereinigung, die beschleunigte Zusammenlegung und den freiwilligen Landtausch nach dem Flurbereinigungsgesetz (FlurbG) ${ }^{6}$, wenn man die städtebaulichen und die fachplanerischländlichen Enteignungsmöglichkeiten sowie einige partielle bundesrechtliche und spezielle landesrechtliche Bodenordnungsvorschriften hier zunächst außer Betracht läßt. Der Einsatz dieser o. g. gesetzlichen Bodenordnungsmaßnahmen ist rechtlich jeweils nur dann zulässig, wenn durch den Einsatz der privatrechtlichen

\footnotetext{
${ }^{5)}$ Baugesetzbuch i. d. F. vom 27. August 1997 (BGB1. I, S. 2141).

${ }^{6)}$ Flurbereinigungsgesetz i. d. F. vom 16. März 19776 (BGB1. I, S. 546), zuletzt geändert durch Gesetz vom 23. August 1994 (BGB1. I, S. 2187).
} 
Bodenordnungsmaßnahmen die Ziele der Bodennutzungsplanung nicht unter angemessenen Bedingungen erreicht werden können - Subsidiaritätsprinzip.

Wirtschaftlich betrachtet ist der Einsatz dieser gesetzlichen Bodenordnungsmaßnahmen ein mit öffentlich-rechtlichen Zwangsmitteln ausgestattetes Grundflächentauschverfahren, weniger ein "Grundstückstauschverfahren". Durch die rechtliche Ausführung von Bodenordnungsplänen treten regelmäßig nach Lage, Beschaffenheit und Nutzbarkeit neue Grundstücke an die Stelle der alten Grundstücke; nur in diesem Sinne wäre es auch ein Tausch. Rechtlich betrachtet wird dem (abstrakt) unveränderten Eigentumsrecht ein neues verändertes Eigentumsobjekt zugeordnet - Surrogationsprinzip.

Im übrigen werden die öffentlich-rechtlichen Bodenordnungsmaßnahmen durch folgende Prinzipien geprägt [3]:

-Die Rechtsverhältnisse der alten Grundstücke werden den zweckdienlichen Maßgaben für die angestrebte Verbesserung der Bodennutzung durch neue Grundstücke angepaßt - Konformitätsprinzip.

-Die Grundflächen für die gemeinschaftlichen und öffentlichen Anlagen werden i. d. R. anteilig von allen Grundeigentümern aufgebracht - Solidaritätsprinzip.

-Das Grundeigentum wird in der Substanz grundsätzlich nicht vermindert und für den jeweiligen Inhaber erhalten - Konservationsprinzip.

-Die Bodenordnung selbst geschieht im wohlverstandenen Interesse der privaten Grundstückseigentümer - Privatnützigkeitsprinzip.

Die regelmäßig nicht objektbezogene Enteignung und in ihrem Vollzuge die städtebauliche Unternehmensflurbereinigung nach dem BauGB sowie die fachplanerisch-ländliche Unternehmensflurbereinigung nach dem FlurbG unterscheiden sich von diesen Bodenordnungsmaßnahmen im engeren Sinne grundsätzlich durch ihr Fremdnützigkeitsprinzip ${ }^{7)}$.

Die öffentlich-rechtliche Bodenordnung (im engeren Sinne) ist Ausfluß der Inhalts- und Schrankenbestimmung nach Artikel 14 Abs. 1 Satz 2 GG. Die Enteignung als Bodenordnungsmaßnahme (im weiteren Sinne) findet ihre Grundlage davon streng getrennt in Artikel 14 Abs. 3 GG. Und eine städtebauliche Umlegung nach dem BauGB bleibt auch dann eine inhaltsbestimmende Maßnahme, wenn sie zu nicht unerheblichen Minderausweisungen, oder sogar dazu führt, daß bestimmte Kleineigentümer kein Land zugeteilt erhalten. Die diesbezüglichen Abfindungsvorschriften sind folglich keine Enteignungsentschädigungsregelungen. Die Vorschriften der $\S \S$ 93 ff. BauGB sind daher auf sie, wie $§ 59$ Abs. 2 Satz 2 BauGB zutreffend sagt, (nur) entsprechend anzuwenden [4]. Für die ländlichen Bodenordnungsmaßnahmen (im engeren Sinne) nach dem FlurbG darf dieser Sachverhalt überhaupt nicht auftreten. Das bedeutet, ein Umschlagen einer öffentlich-rechtlichen Bodenordnung (im engeren Sinne) in eine Enteignung als Bodenordnungsmaßnahme (im weiteren Sinne) ist regelmäßig undenkbar; diese Bo-

\footnotetext{
${ }^{7)}$ Bundesverfassungsgericht-Urt. v. 24. März 1987 - 1 BvR - 1046/85; NJW 1987, S. 1251 = AgrarR 1987, S. 190.
}

denordnungsmaßnahme wird ggf. rechtlich dann wohl unzulässig.

\section{Zum System der Bodennutzungsplanung}

Die wesentlichen Grundlagen der Bodennutzungsplanung in der Bundesrepublik Deutschland sind im Bundesraumordnungsgesetz $(\mathrm{ROG})^{8)}$, in den jeweiligen Landesplanungsgesetzen $(\mathrm{LaPlaG})^{9)}$ sowie im BauGB dargestellt; sie werden durch die verschiedenen Fachplanungsgesetze des Bundes und der Länder ergänzt. Gegliedert ist dieses Planungssystem in (Abb. 1):

- die obere Ebene der Raumordnung des Bundes,

- die mittlere Ebene von Raumordnung, Landesplanung und Regionalplanung der Länder,

- die untere Ebene der Bauleitplanung der Gemeinden.

Die spezialbehördliche Fachplanung erfolgt zwischen diesen Planungsebenen. Insgesamt prägt dabei der Träger der jeweils niedrigeren Ebene die Planung auf der jeweils höheren Ebene im sog. "Gegenstromverfahren" mit.

Das Raumordnungsgesetz enthält naturgemäß nur abstrakt formulierte Aufgaben, Leitvorstellungen und Grundsätze. Danach sind der Gesamtraum der Bundesrepublik Deutschland und seine Teilräume durch zusammenfassende, übergeordnete Raumordnungspläne und durch Abstimmung raumbedeutsamer Planungen und Maßnahmen zu entwickeln, zu ordnen und $\mathrm{zu}$ sichern. Dabei sind die unterschiedlichen Anforderungen an den Raum aufeinander abzustimmen und die auf der jeweiligen Planungsebene auftretenden Konflikte auszugleichen und Vorsorge für die einzelnen Raumfunktionen und Raumnutzungen zu treffen ( 1 Abs. 1 ROG).

Leitvorstellung bei der Erfüllung dieser Aufgabe ist eine nachhaltige Raumentwicklung, die die sozialen und wirtschaftlichen Ansprüche an den Raum mit seinen ökologischen Funktionen in Einklang bringt und zu einer dauerhaften, großräumig ausgewogenen Ordnung führt; dabei sind

-die freie Entfaltung der Persönlichkeit in der Gemeinschaft und in der Verantwortung gegenüber künftiger Generationen zu gewährleisten,

- die natürlichen Lebensgrundlagen zu schützen und zu entwickeln,

- die Standortvoraussetzungen für die wirtschaftlichen

Entwicklungen zu schaffen,

-die Gestaltungsmöglichkeiten der Raumnutzung langfristig offen zu halten,

- die prägende Vielfalt der Teilräume zu stärken,

- gleichwertige Lebensverhältnisse in allen

Teilräumen herzustellen,

-die räumlichen und strukturellen Ungleichgewichte zwischen den bis zur Herstellung der Einheit Deutschlands getrennten Gebieten auszugleichen und

-die räumlichen Voraussetzungen für den Zusammenhalt in der Europäischen Gemeinschaft und im größeren europäischen Raum zu schaffen.

\footnotetext{
${ }^{8)}$ Raumordnungsgesetz vom 18. August 1997 (BGB1. I, S. 2081).

9) z. B. im Bundesland Nordrhein-Westfalen, Landesplanungsgesetz i. d.

F. vom 29. Juni 1994 (GB. NW. S. 474).
} 


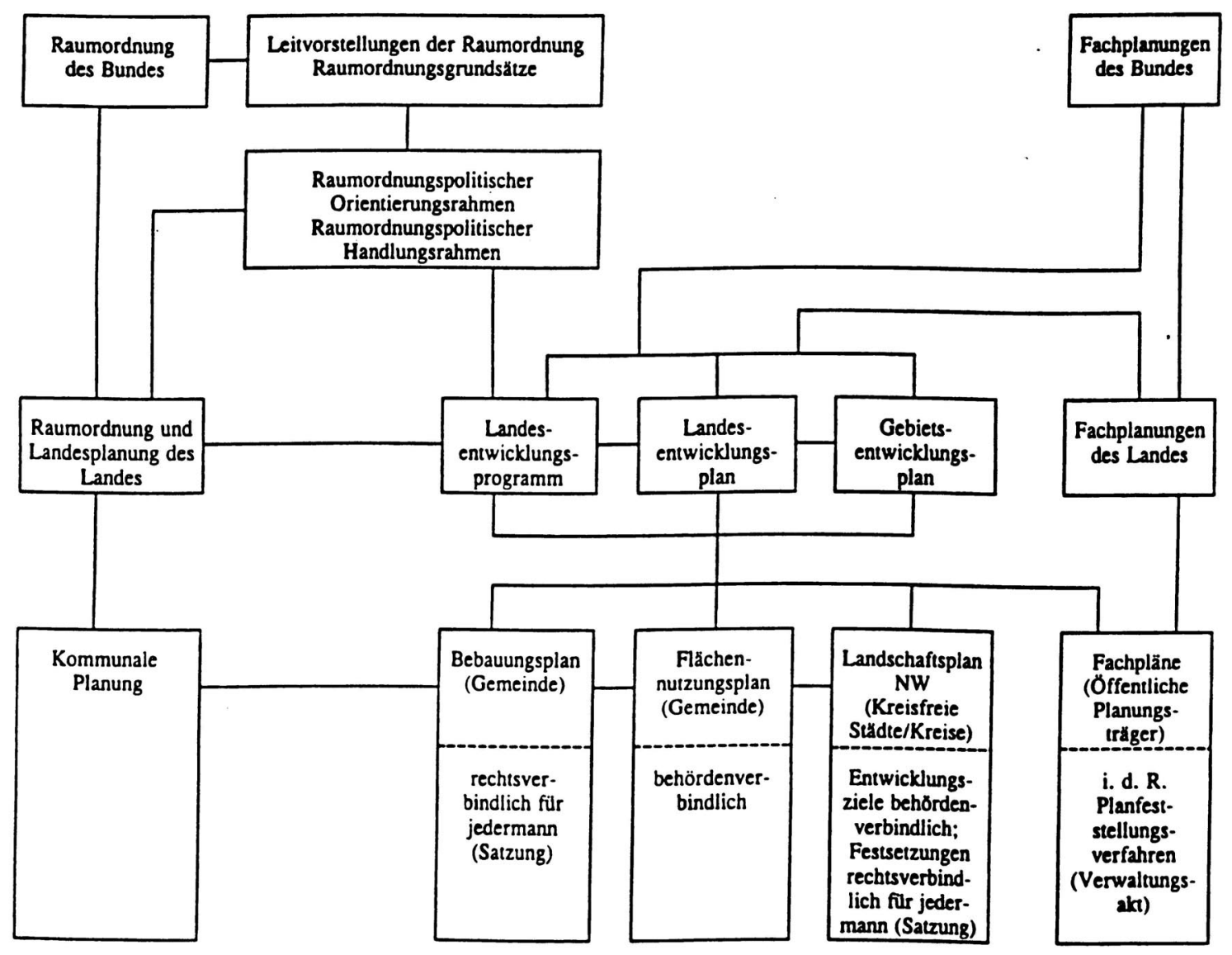

Abbildung 1. Wirkungsgefüge wesentlicher Sach- und Fachzusammenhänge der Bodennutzungsplannung in Deutschland unter besonderer berïcksichtigung des Landes Nordrhein-Westfalen.

Quelle: Linke, H.-J. (1996), Zur Harmonisierung der Grundstücksneuordnungsinstrumentarien, Dissertation Universität Bonn

Dabei soll die Entwicklung, Ordnung und Sicherung der Teilräume sich in die Gegebenheiten und Erfordernisse des Gesamtraumes einfügen; die Entwicklung, Ordnung und Sicherung des Gesamtraumes soll zugleich die Gegebenheiten und Erfordernisse seiner Teilräume berücksichtigen ( $\$ 1$ Abs. 2 und 3 ROG).

Inhaltlich und räumlich konkretisiert werden diese Vorgaben der Raumordnung des Bundes durch die Raumordnung und Landesplanung des jeweiligen Bundeslandes. Deren Entwicklungsprogramme und Entwicklungspläne stellen die Richtlinien für die nachgeordneten Planungsebenen dar. Raumordnung und Landesplanung sind dabei räumlich und sachlich umfassend. Sie beziehen sich jeweils auf das gesamte Bundes- bzw. Landesgebiet und beinhalten alle planerisch bedeutsamen Sachverhalte. Noch weiter konkretisiert, spezifiziert und lokalisiert werden diese übergeordneten und überörtlichen Planungen durch die Bauleitplanung der Gemeinden und die sektoral sehr differenzierten Fachplanungen.

Die Verwirklichung der verbindlichen Bauleit- und Fachplanungen bleibt bezüglich der privaten Nutzung grundsätzlich dem Grundeigentümer überlassen. Nur ausnahmsweise kann die geplante private Nutzung hoheitlich erzwungen werden, z. B. durch Bau- oder Nutzungsgebote. Indes sind die jeweiligen Planungsträger, wie der Bund, die Bundesländer und die Gemeinden grundsätzlich gehalten, die verbindlich geplante Nutzung unter angemessenen Bedingungen zu ermöglichen.

So obliegt der Gemeinde die Pflicht, neue Baugebiete $\mathrm{zu}$ erschließen, d. h. alle verkehrlichen und versorgungstechnischen Voraussetzungen zu schaffen, die für die Nutzung von Gebäuden und sonstigen plangemäßen Grundstückseinrichtungen erforderlich sind. Um die Erschließung und plangemäße Bebauung zu ermöglichen, sind erforderlichenfalls die betroffenen Grundstücke nach Lage, Form und Größe zweckmäßig zu gestalten, also städtebauliche Bodenordnungsmaßnahmen, wie Umlegung (nach den $\S \S 45$ ff. BauGB) und Grenzregelung (nach den $\S \S 80 \mathrm{ff}$. BauGB) oder mittels städtebaulichem Enteignungsrecht als Entwicklungsmaßnahme (nach den $\S \S 165$ ff. BauGB) und Unternehmensflurbereinigung (nach den $\$ \S 190$ ff. BauGB) einzuleiten. Und sofern diese Bodenordnungsmaßnahmen nicht vom Grundstückseigentümer selbst bewerkstelligt werden können, obliegen sie nach dem Subsidaritätsprinzip der Gemeinde.Anzumerken bleibt hier, daß für bestimmte fremdnützige städtebauliche, also enteignungsrechtliche Bodenordnungsprobleme kein adäquates Bodenordnungsinstrument zur Verfügung steht, da die Umlegung und Grenzregelung grundsätzlich privatnützig ausgerichtet 
sind und die städtebauliche Entwicklungsmaßnahme in Stadt und Land nur eingesetzt werden kann, wenn deren einheitliche Vorbereitung und zügige Durchführung im öffentlichen Interesse liegen; bei städtebaulichen Unternehmensflurbereinigungen wird bisher immer einschränkend die Inanspruchnahme land- und/oder forstwirtschaftlicher Grundstücke vorausgesetzt, ansonsten ist dieses fremdnützige und in der allgemeinen Fachplanung sehr bewährte Bodenordnungsinstrument hier bisher nicht zulässig (man vergleiche das Problem Winterberg) [5]. Bei ggf. erweitertem Anwendungsbereich wäre die nur fingierte Privatnützigkeit mancher Umlegung obsolet.

Im Sinne der vorstehend genannten Leitvorstellungen der Raumordnung ( $\$ 1$ Abs. 2 und 3 ROG) sind im wesentlichen folgende Grundsätze der Raumordnung zur nachhaltigen Raumentwicklung anzuwenden ( $\$ 2$ Abs. 1 und 2 ROG):

-Im Gesamtraum der Bundesrepublik Deutschland ist eine ausgewogene Siedlungs- und Freiraumstruktur $\mathrm{zu}$ entwickeln.

-Die dezentrale Siedlungsstruktur des Gesamtraumes mit ihrer Vielzahl leistungsfähiger Zentren und Stadtregionen ist zu erhalten.

-Die großräumige und übergreifende Freiraumstruktur ist zu erhalten und zu entwickeln.

-Die Infrastruktur ist mit der Siedlungs- und Freiraumstruktur in Übereinstimmung zu bringen.

- Verdichtete Räume sind als Wohn-, Produktionsund Dienstleistungsschwerpunkte zu sichern.

-Ländliche Räume sind als Lebens- $u$. Wirtschaftsräume mit eigenständiger Bedeutung $\mathrm{zu}$ entwickeln.

$-\mathrm{Zu}$ einer räumlich ausgewogenen, langfristig wettbewerbsfähigen Wirtschaftsstruktur sowie $\mathrm{zu}$ einem ausreichenden und vielfältigen Angebot an Arbeits- und Ausbildungsplätzen ist beizutragen.

-Es sind die räumlichen Voraussetzungen dafür zu schaffen oder $\mathrm{zu}$ sichern, daß die Landwirtschaft als leistungsfähiger Wirtschaftszweig sich dem Wettbewerb entsprechend entwickeln kann und gemeinsam mit einer leistungsfähigen Forstwirtschaft dazu beiträgt, die natürlichen Lebensgrundlagen zu schützen sowie Natur und Landschaft zu pflegen und zu gestalten.

-Dem Wohnraumbedarf der Bevölkerung ist Rechnung zu tragen; dabei ist die Eigenentwicklung der Gemeinden bei der Wohnraumversorgung ihrer Bevölkerung zu gewährleisten.

-Eine gute Erreichbarkeit aller Teilräume untereinander durch Personen- und Güterverkehr ist sicherzustellen.

-Die geschichtlichen und kulturellen Zusammenhänge sowie die regionale Zusammengehörigkeit sind zu wahren; dabei sind die gewachsenen Kulturlandschaften mit ihren prägenden Merkmalen sowie mit ihren Natur- und Kulturdenkmäler zu sichern.

-Für Erholung in Natur und Landschaft sowie für Freizeit und Sport sind geeignete Gebiete und Standorte zu sichern.
-Den räumlichen Erfordernissen der zivilen und militärischen Verteidigung ist Rechnung zu tragen.

Um diese nachhaltige Raumentwicklung vor allem in den ländlichen Gebieten und am Rande der städtischen Verflechtungsgebiete in der Bundesrepublik Deutschland verwirklichen zu können, haben die jeweiligen Organe der Gesetzgebung, der planenden Verwaltung und der Rechtsprechung in einer jahrzehntewährenden Entwicklung das Flurbereinigungsgesetz $\mathrm{zu}$ einem vielseitigen Fachplanungsinstrument ausgestaltet. Flurbereinigung bedeutet dabei heute allgemein, eine Neuordnung ländlichen Grundbesitzes durch Maßnahmen zur Verbesserung der Produktions- und Arbeitsbedingungen in der Land- und Forstwirtschaft sowie zur Förderung der allgemeinen Landeskultur und der Landentwicklung (§ 1 FlurbG).

Die Verbesserung der Produktions- und Arbeitsbedingungen in der Land- und Forstwirtschaft der Bundesrepublik Deutschland hat sich dabei an der Wirtschaftlichkeit und Wettbewerbsfähigkeit der land- und forstwirtschaftlichen Betriebe, d. h. ihrer Produktivität, zu orientieren. Die Förderung der allgemeinen Landeskultur umfaßt dabei alle Maßnahmen zur Verbesserung der Agrarstruktur und zur Landschaftspflege unter Berücksichtigung der ökologischen Ausgleichsfunktion der ländlichen Gebiete. Und die Förderung der Landentwicklung umfaßt alle Maßnahmen des Planens, Vorbereitens und Verwirklichens, die geeignet sind, die Wirtschafts-, Wohn- und Erholungsfunktionen in den ländlichen Gebieten und am Rande der städtischen Verflechtungsgebiete $\mathrm{zu}$ erhalten und $\mathrm{zu}$ verbessern, um damit für eine dauerhafte Verbesserung der Lebensverhältnisse außerhalb der städtischen Gebiete zu sorgen.

Zur eigentumsrechtlichen Verwirklichung aller sonstigen grundeigentumsbezogenen Fachplanungen in der Bundesrepublik Deutschland, wie

- der bundesfernstraßenrechtlichen Planfeststellung,

- der eisenbahnrechtlichen Planfestellung,

- der luftverkehrsrechtlichen Planfeststellung,

- der bundeswasserstraßenrechtlichen Planfeststellung,

-der personenbeförderungsrechtlichen Planfeststellung,

-der wasserrechtlichen (wasserwirtschaftlichen)

Planfeststellung,

- der abfallentsorgungsrechtlichen Planfeststellung,

- der energiewirtschaftlichen Planfeststellung,

- der fernmelderechtlichen Planfeststellung,

- der bergrechtlichen Planfeststellung u. v. a. m.

wird regelmäßig auf die Enteignung als öffentlichrechtliche Bodenordnungsmaßnahme (im weiteren Sinne) verwiesen. Nach dem allgemeinen Verwaltungsgrundsatz der Verhältnismäßigkeit staatlichen Handelns greift hier das Instrument der fachplanerisch-ländlichen Unternehmensflurbereinigung (nach den $\$ \S 87$ ff. FlurbG). Dieses gewährleistet nach der Konfliktbewältigung im jeweiligen Planaufstellungs- bzw. Planfeststellungsverfahren den schonenden Umgang mit dem Grundeigentum der Betroffenen [6]. 


\section{Die neugestaltenden öffentlich-rechtlichen Bodenordnungsinstrumente}

\subsection{Zur Umlegung und Grenzregelung nach dem Allgemeinen Städtebaurecht des Baugesetzbuches}

Eine Gemeinde kann durch Umlegung im Geltungsbereich eines Bebauungsplanes und innerhalb der im Zusammenhang bebauten Ortsteile zur Erschließung und/oder zur Neugestaltung bestimmter Gebiete bebaute und unbebaute Grundstïcke neu ordnen, damit nach Lage, Form und Größe für die bauliche oder die sonstige städtebaulich motivierte Nutzung zweckmäßig gestaltete Grundstücke entstehen. Innerhalb der im Zusammenhang bebauten Ortsteile kann eine Umlegung durchgeführt werden, wenn sich aus der Eigenart der näheren
Umgebung hinreichende Kriterien für die Neuordnung der Grundstücke ergeben. ( $\$ 45$ Abs. 1 BauGB).

Ein qualifizierter Bebauungsplan liegt für die Gemeinde (als Satzung) vor, wenn er allein oder gemeinsam mit sonstigen baurechtlichen Vorschriften mindestens Festsetzungen über die Art und das Maß der baulichen Nutzung, die überbaubaren Grundstücksflächen und die örtlichen Verkehrsflächen enthält. Hier ist ein Vorhaben zulässig, wenn es diesen Festsetzungen nicht widerspricht und die Erschließung gesichert ist. Ein Bebauungsplan, der diese Mindestfestsetzungen nicht enthält, gilt als einfacher Bebauungsplan. Die Zulässigkeit von Vorhaben richtet sich in diesem Falle im übrigen nach den Vorschriften über die im Zusammenhang bebauten Ortsteile oder das Bauen im Außenbereich (§ 30 Abs. 1 und 3 BauGB).

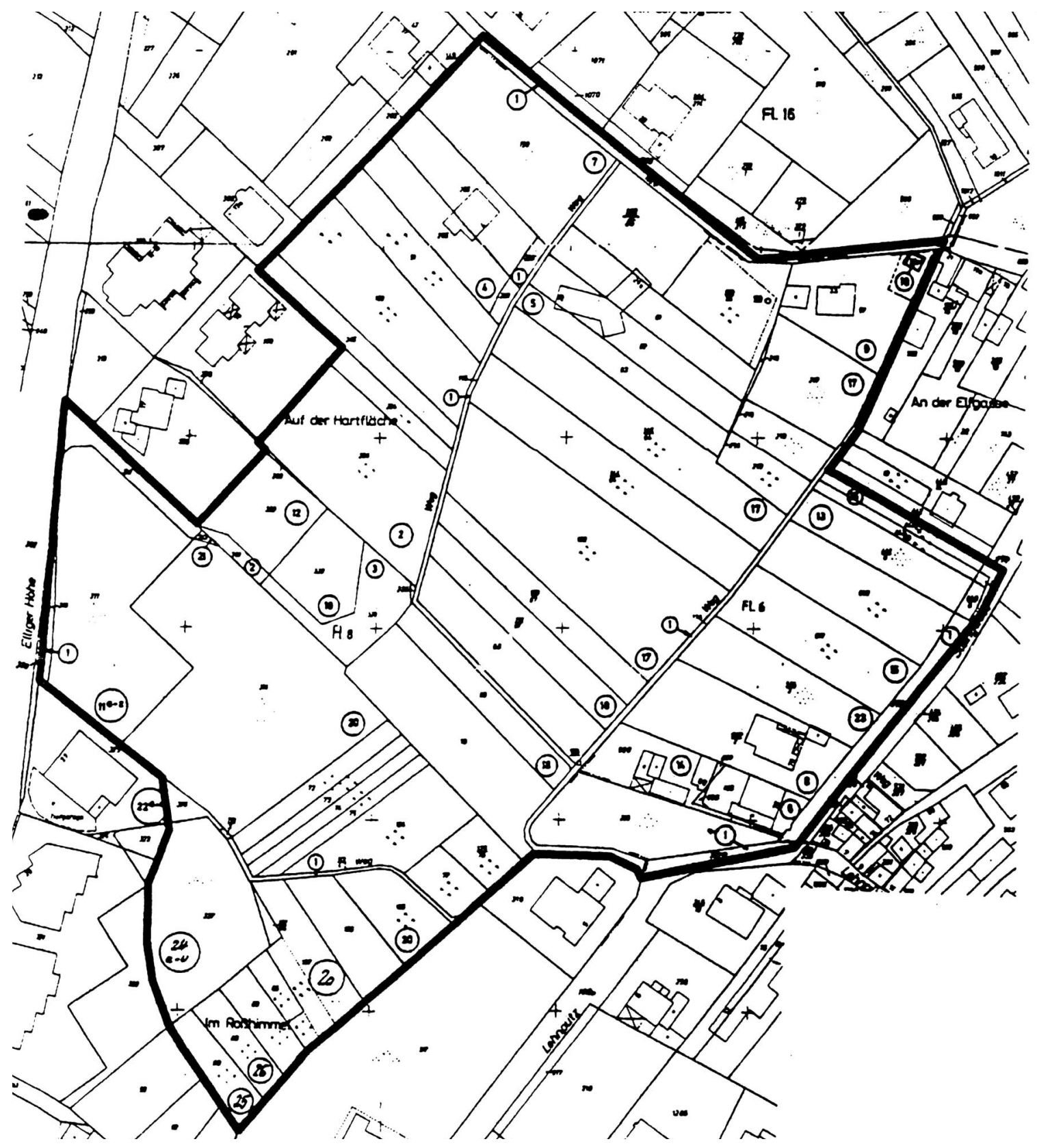

Abbildung 2. Karte des alten Bestandes 


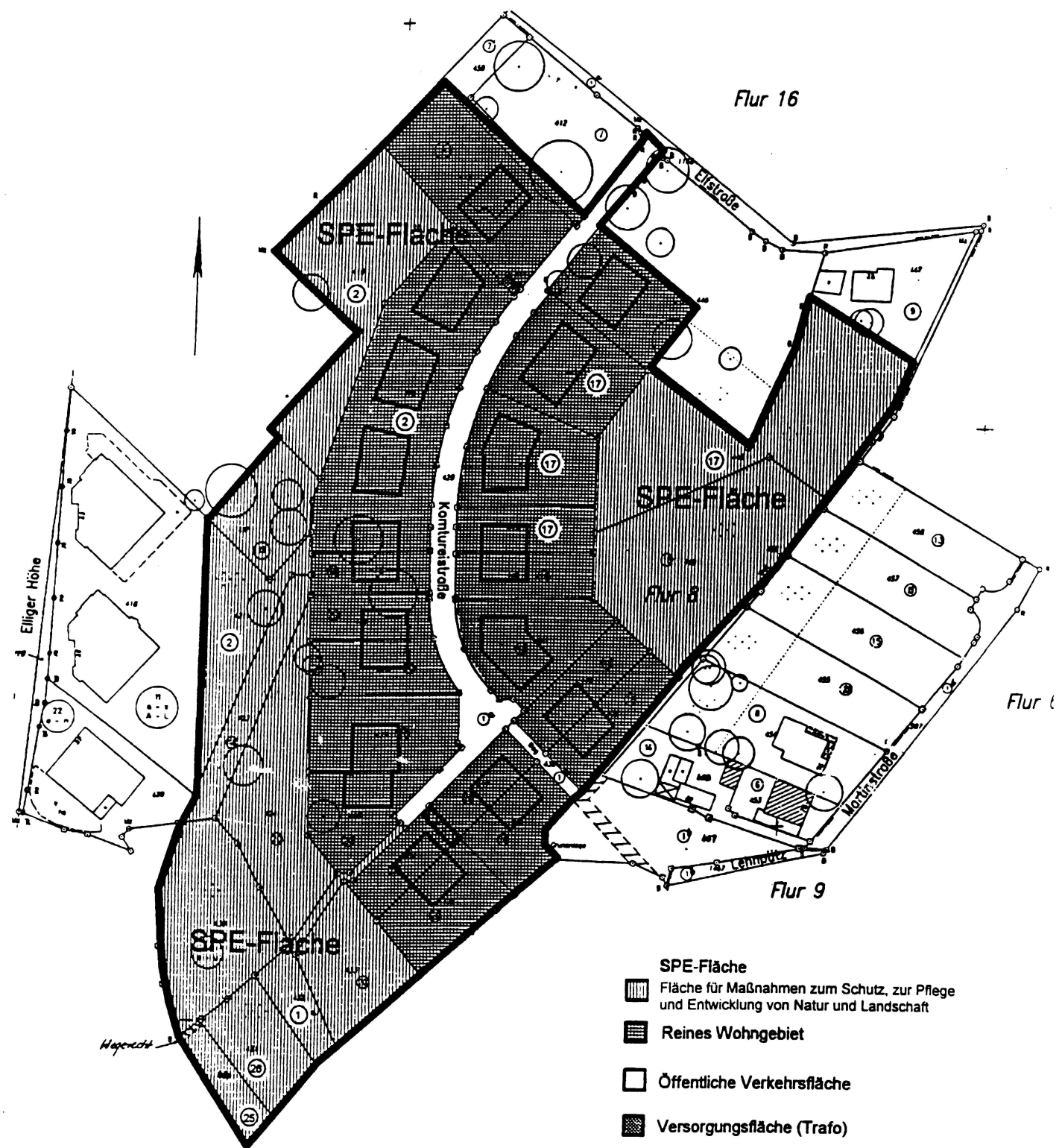

Abbildung 3. Karte des neuen Bestandes (1. Teilplan). Hier gelten die jeweiligen Landesvorschriften zum Vermessungs- und Katasterrecht

Wegen seiner zunehmenden Bedeutung ist in diesem Zusammenhang auch auf das ergänzende Satzungsrecht der Gemeinden hinzuweisen; sie kann damit

- die Grenzen für im Zusammenhang bebaute Ortsteile festlegen

-die Abgrenzungssatzung (nach § 34 Abs. 4 Nr. 1 BauGB) -

-bebaute Bereiche im Außenbereich als im Zusammenhang bebaute Ortsteile festlegen, wenn die Flächen bereits im vorbereitenden Bebauungsplan (Flächennutzungsplan) als Baufläche dargestellt sind

-die Entwicklungssatzung (nach $§ 34$ Abs. 4 Nr. 2 BauGB) - und

- einzelne Außenbereichsflächen in die im Zusammenhang bebauten Ortsteile einbeziehen, wenn die einbezogenen Flächen durch die bauliche Nutzung als angrenzende Bereiche entsprechend geprägt sind

-die Abrundungssatzung (nach § 34 Abs. 4 Nr. 3 BauGB).
Eine Gemeinde kann durch Grenzregelung im Geltungsbereich eines Bebauungsplanes oder innerhalb der im Zusammenhang bebauten Ortsteile zur Herbeiführung einer ordnungsgemäßen Bebauung einschließlich Erschließung und/oder zur Beseitigung baurechtswidriger Zustände (Abbildungen 2, 3).

- benachbarte Grundstücke oder Teile benachbarter Grundstücke gegeneinander austauschen, wenn dies dem überwiegenden öffentlichen Interesse dient oder

-benachbarte Grundstücke, insbesondere Splittergrundstücke oder Teile benachbarter Grundstücke einseitig zuteilen, wenn dies im öffentlichen Interesse geboten ist.

Die Grundstücke und Grundstücksteile dürfen nicht selbständig bebaubar und eine durch die Grenzregelung für den Grundstückseigentümer bewirkte Wertminderung darf nur unerheblich sein ( $\$ 80$ Abs. 1 BauGB). 
4.2. Zur Flurbereinigung, vereinfachten Flurbereinigung, beschleunigten Zusammenlegung und zum freiwilligen Landtausch nach dem Fachplanungsrecht des Flurbereinigungsgesetzes

Die Flurbereinigungsverwaltung eines Bundeslandes kann ein Bodenordnungsverfahren nach dem FlurbG einleiten, um die Produktions- und Arbeitsbedingungen in der Land- und Forstwirtschaft $\mathrm{zu}$ verbessern und die allgemeine Landeskultur und die Landentwicklung $\mathrm{zu}$ fördern (Programmsatz der Flurbereinigung (nach $§ 1$ FlurbG)).

Daraus erwachsen die allgemeinen Aufgaben der Flurbereinigung im engeren Sinne ( $\$ 37$ Abs. 1 FlurbG):

-Das jeweilige Flurbereinigungsgebiet ist unter Beachtung der vorhandenen Landschaftsstruktur neu $\mathrm{zu}$ gestalten, wie es den gegeneinander abzuwägenden Interessen der Beteiligten sowie den Interessen der allgemeinen Landeskultur und der Landentwicklung entspricht und wie es das Wohl der Allgemeinheit erfordert.

-Die Feldmark ist neu einzuteilen und zersplitterter oder unwirtschaftlich geformter Grundbesitz ist nach neuzeitlichen betriebswirtschaftlichen Gesichtspunkten zusammenzulegen und nach Lage, Form und Größe zweckmäßig zu gestalten (Abb. 4).

-Wege, Straßen, Gewässer und andere gemeinschaftliche Anlagen sind zu schaffen.
-Bodenverbessernde, bodenschützende und landschaftsgestaltende Maßnahmen sind vorzunehmen.

-Die rechtlichen Verhältnisse sind zu ordnen.

- Alle sonstigen Maßnahmen zur Verbesserung der Grundlagen der land- und forstwirtschaftlichen Betriebe, zur Verminderung des Arbeitsaufwandes und zur Erleichterung der Bewirtschaftung sind vorzunehmen.

-Maßnahmen der Dorferneuerung können durchgeführt werden; dabei wird die Zuziehung von Ortslagen zur Flurbereinigung durch Bebauungspläne und ähnliche Planungen nicht ausgeschlossen.

Diese allgemeinen Aufgaben werden durch folgende besondere Aufgaben der Flurbereinigung im engeren Sinne ergänzt ( $\$ 86, \S 91$ und $\S 103$ a FlurbG):

- $\quad$ Ein vereinfachtes Flurbereinigungsverfahren kann danach eingeleitet werden, um

1. Maßnahmen der Landentwicklung, insbesondere Maßnahmen der Agrarstrukturverbesserung, der Siedlung, der Dorferneuerung, städtebauliche Maßnahmen, Maßnahmen des Umweltschutzes, der naturnahen Entwicklung von Gewässern, des Naturschutzes und der Landschaftspflege oder der Gestaltung des Orts- und Landschaftsbildes zu ermöglichen oder auszuführen,

2. Nachteile für die allgemeine Landeskultur $\mathrm{zu}$ beseitigen, die durch Herstellung, Änderung oder Beseitigung von Infrastrukturanlagen oder durch ähnliche Maßnahmen entstehen oder entstanden sind,

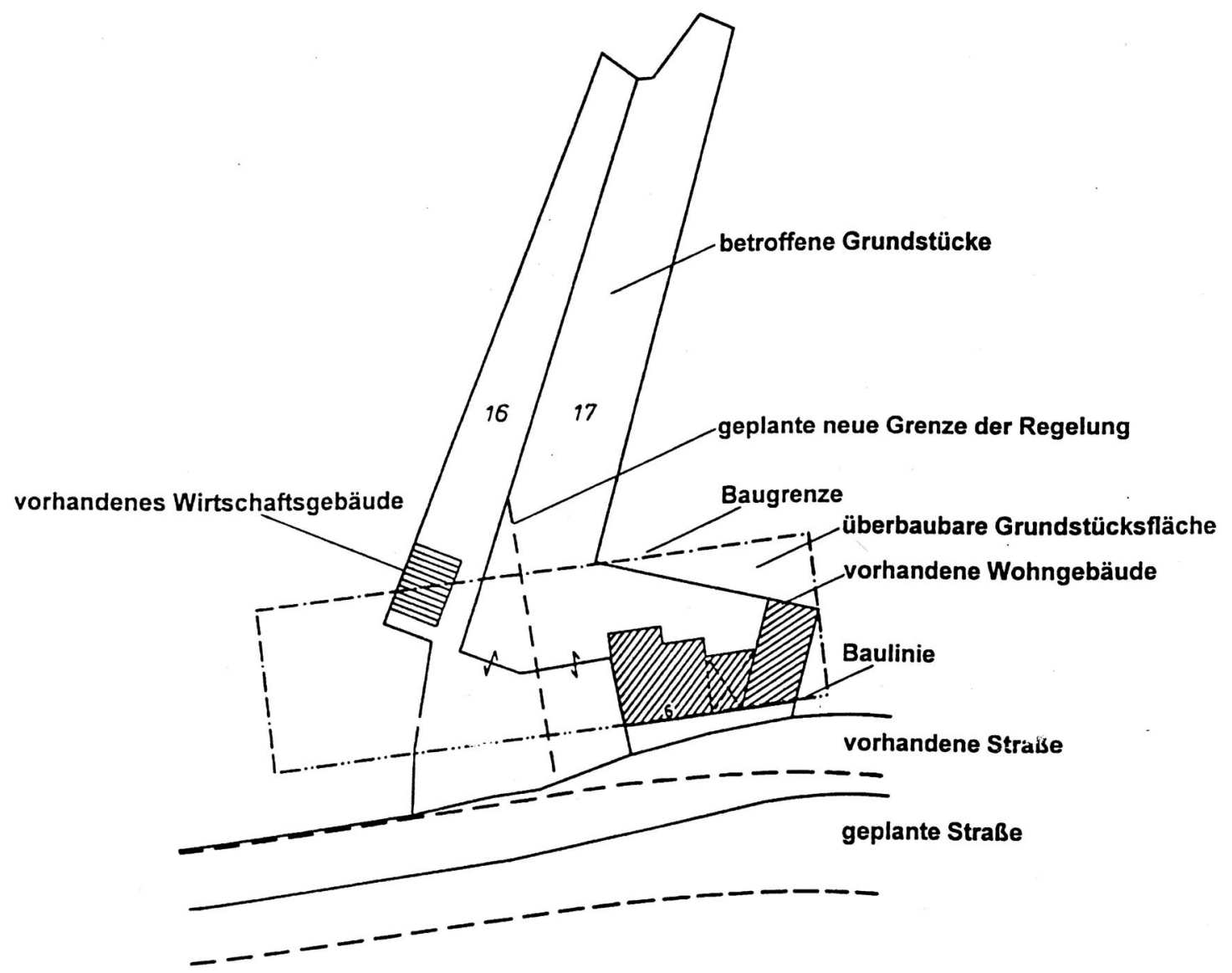

Abbildung 4. Prinzipdarsellung einer Grenzregelung für zwei betroffene Grundstücke Quelle: Professur für Bodenordnung und Bodenwirtschaft der Universität Bonn (1990) 
3. Landnutzungskonflikte aufzulösen oder

4. eine erforderlich gewordene Neuordnung des Grundbesitzes in Weilern, Gemeinden kleineren Umfanges, Gebieten mit Einzelhöfen sowie in bereits flurbereinigten Gemeinden durchzuführen.

- Ein beschleunigtes Zusammenlegungsverfahren kann in den Gemarkungen stattfinden, in denen die Anlage eines neuen Wegenetzes und größere wasserwirtschaftliche Maßnahmen zunächst nicht erforderlich sind, um

1. die in der allgemeinen Flurbereinigung angestrebte Verbesserung der Produktions- und Arbeitsbedingungen in der Land- und Forstwirtschaft möglichst rasch herbeizuführen oder

2. um notwendige Maßnahmen des Naturschutzes und der Landschaftspflege zu ermöglichen. werden

-Ein freiwilliger Landtausch kann durchgeführt

1. um ländliche Grundstücke zur Verbesserng der Agrarstruktur in einem schnellen und einfachen Verfahren neu zu ordnen oder

2. aus Gründen des Naturschutzes und der Landschaftspflege.

Bei der Verwirklichung dieses umfangreichen Kataloges der Flurbereinigungsaufgaben im engeren Sinne haben die Flurbereinigungsverwaltungen ergänzend einen Rahmen der Flurbereinigungsaufgaben im weiteren Sinne zu beachten ( $\$ 37$ Abs. 2 FlurbG), indem sie die öffentlichen Interessen wahren und den Erfordernissen der Raumordnung, der Landesplanung und einer geordneten städtebaulichen Entwicklung, des Umweltschutzes, des Naturschutzes und der Landschaftspflege, des Denkmalschutzes, der Erholung, der Wasserwirtschaft einschließlich der Wasserversorgung und Abwasserbeseitigung, der Fischerei, des Jagdwesens, der Energieversorgung, des öffentlichen Verkehrs, der landwirtschaftlichen Siedlung, der Kleinsiedlung, des Kleingartenwesens und der Gestaltung des Orts- und Landschaftsbildes sowie einer möglichen bergbaulichen Nutzung und der Erhaltung und Sicherung mineralischer Rohstoffvorkommen Rechnung tragen.

"Rechnung tragen" heißt dabei nach einem Urteil des Oberverwaltungsgerichts Münster ${ }^{10)}$ je nach Lage des Einzelfalles die vorstehend genannten öffentlichen Belange dann mitzuberücksichtigen und entsprechende Planungen anderer ganz oder teilweise mitzuverwirklichen, wenn dabei gleichwohl eine im Sinne des Flurbereinigungsgesetzes gerechte Abfindung aller Beteiligten des Flurbereinigungsverfahrens möglich bleibt, und wenn dadurch der Ablauf des Flurbereinigungsverfahrens nicht oder nur unwesentlich verzögert wird.

Allgemein tendiert die Entwicklung der Flurbereinigungsaufgaben heute weg von den großen und komplexen Aufgabenstrukturen hin zu kleineren übersichtlich strukturierten Einzelaufgaben. Nicht ganz unproblematisch ist in diesem Zusammenhang die absolut anerkennenswerte bodenordnerische Verwirklichung umfangreicher Naturschutz- und Landschaftsplanungen als eigenständige Fachplanungen, denn auch sie sind in aller Regel überwiegend fremdnützig; sie werden aber dennoch in den besonderen Bodenordungsverfahren nach dem Flurbereinigungsgesetz ermöglicht, die hier ausschließlich dem Prinzip der Privatnützigkeit unterliegen. Entsprechendes gilt selbstverständlich auch für die Auflösung fremdnützig verursachter Landnutzungskonflikte anderer Art [7].

\subsection{Zur fachplanerisch-ländlichen und zur städtebaulichen Unternehmensflurbereinigung nach dem Flurbereinigungsgesetz bzw. nach dem Baugesetzbuch}

Die Flurbereinigungsverwaltung eines Bundeslandes kann auf Antrag der jeweiligen Enteignungsbehörde eine fachplanerisch-ländliche Unternehmensflurbereinigung einleiten, wenn aus besonderem Anlaß eine fachplanerische Enteignung zulässig ist, durch die ländliche Grundstücke in großem Umfang in Anspruch genommen werden und der den Betroffenen entstehende Landverlust auf einen größeren Kreis von Eigentümern verteilt und/oder Nachteile für die allgemeine Landeskultur, die durch das Unternehmen entstehen, vermieden werden sollen ( $\$ 87$ Abs. 1 Satz 1 FlurbG).

Eine städtebauliche Unternehmensflurbereinigung kann auf Antrag einer Gemeinde bei Zustimmung der höheren Verwaltungsbehörde eingeleitet werden, wenn durch städtebauliche (Enteignungs-) Möglichkeiten landund/oder forstwirtschaftliche Grundstücke in Anspruch genommen werden, damit die den Betroffenen dabei entstehenden Landverluste auf einen größeren Kreis von Eigentümern verteilt und/oder dabei entstehende Nachteile für die allgemeine Landeskultur vermieden werden können. Der Träger des Unternehmens wäre in diesem Falle die Gemeinde. (Nach $\S 190$ Abs. 1 BauGB).

Im Detail sind dabei folgende wesentliche Aspekte hervorzuheben:

-Die für das Unternehmen benötigten Flächen sind, soweit sie nicht vorab freihändig erworben werden konnten, von den Teilnehmern nach dem Verhältnis des Wertes ihrer alten Grundstücke zu dem Wert aller Grundstücke des Verfahrensgebietes aufzubringen. Für diese von den Teilnehmern aufgebrachten Flächen hat ihnen der Träger des Unternehmens Geldentschädigung zu leisten.

-Der Träger des Unternehmens hat Nachteile, die den Beteiligten durch das Unternehmen entstehen, zu beheben und, soweit dies nicht möglich ist oder nach dem Ermessen der Flurbereinigungsbehörde nicht zweckmäßig erscheint, für sie Geldentschädigung zu leisten.

-Für die jeweiligen Geldentschädigungen gilt das Recht des Unternehmens.

-Der Träger des Unternehmens hat die von ihm verursachten Ausführungs- und Verfahrenskosten der Unternehmensflurbereinigung zu tragen.

\footnotetext{
${ }^{10)}$ OVG Münster, Urt. vom 21. Nov. 1968; in: Recht der Landwirtschaft
} (RdL) 1969, S. 272 ff. 


\subsection{Die städtebauliche Entwicklungsmaßnahme nach dem Besonderen Städtebaurecht des Baugesetzbuches}

Mit städtebaulichen Entwicklungsmaßnahmen sollen Ortsteile und andere Teile des Gemeindegebietes entsprechend ihrer besonderen Bedeutung für die städtebauliche Entwicklung und Ordnung der Gemeinde oder entsprechend der angestrebten Entwicklung des Landesgebietes oder der Region erstmalig entwickelt oder im Rahmen einer städtebaulichen Neuordnung einer neuen Entwicklung zugeführt werden ( $\$ 165$ Abs. 2 BauGB).

Die Gemeinde kann einen Bereich, in dem eine städtebauliche Entwicklungsmaßnahme durchgeführt werden soll, durch Beschluß förmlich als städtebaulichen Entwicklungsbereich festlegen, wenn

1. die Maßnahme den vorstehend genannten Zielen und Zwecken entspricht,

2. das Wohl der Allgemeinheit die Durchführung der städtebaulichen Entwicklungsmaßnahme erfordert, insbesondere zur Deckung eines erhöhten Bedarfs an Wohn- und Arbeitsstätten, zur Errichtung von Gemeinbedarfs- und Folgeeinrichtungen oder zur Wiedernutzung brachliegender Flächen,

3. die mit der städtebaulichen Entwicklungsmaßnahme angestrebten Ziele und Zwecke durch städtebauliche Verträge nicht erreicht werden können oder Eigentümer der von der Maßnahme betroffenen Grundstücke nicht bereit sind, ihre Grundstïcke an die Gemeinde oder den von ihr beauftragten Entwicklungsträger $\mathrm{zu}$ angemessenen Bedingungen zu veräußern,

4. die zügige Durchführung der Maßnahme innerhalb eines absehbaren Zeitraumes gewährleistet ist.

Die öffentlichen und die privaten Belange sind dabei gerecht gegeneinander und untereinander abzuwägen $(\S$ 165 Abs. 3 BauGB).

Besonders beachtlich ist hierbei das Erfordernis des Wohls der Allgemeinheit, also die enteignungsrechtliche Grundlage nach Artikel 14 Abs. 3 GG, die in der anfänglichen Praxis nicht immer deutlich wurde. Außerdem sollte der kombinierte Einsatz dieses städtebaulichen Bodenordnungsinstruments mit der städtebaulichen Unternehmensflurbereinigung, wie er im ursprünglichen Städtebauförderungsgesetz des Jahres 1971 angedacht war, einmal erprobt werden.

\section{Resümé}

Die vielschichtige und vielgestaltige Bodennutzungsplanung in der Bundesrepublik Deutschland gewährleistet regelmäßig für alle Bereiche unseres Gemeinwesens hinreichende Gestaltungsmöglichkeiten - ein gesondertes Problem in städtischen Verflechtungs-gebieten bzw. am Stadtrand trat bisher nicht auf. Für die naturgemäß auftretenden Interessenverflech-tungen und Interessengegensätze stehen vielfältige Mechanismen der Konfliktbewältigung zur Verfügung. Die Qualität der Planung bleibt natürlich insofern abhängig von der sorgfältigen Arbeit der Konfliktbewältiger. Ihre sinnvolle Ausgestaltung schützt in hohem Maße vor Fehlplanungen und damit vor Fehlinvestitionen und letztendlich vor volkswirtschaftlichen Schäden.
Bezüglich der eigentumsrechtlichen Konfliktbewältigung bei den jeweils betroffenen Grundeigentümern stehen ebenfalls vielschichtig und vielgestaltig wirkende Instrumentarien zur Verfügung. Dennoch - letztendlich ist jeweils nur ein ganz bestimmtes Bodenordnungsverfahren rechtlich zulässig; es besteht keine freie Wahl der Instrumente, denn dieses würde Behördenwillkür manifestieren und verstieße damit gegen das Rechtsstaatsprinzip des Grundgesetzes der Bundesrepublik Deutschland. Dieses möge man auch bei gelegentlichen politischen Maßgaben zur Gesetzesanwendung beachten.

Bodenordnungsmaßnahmen bedingen immer auch eine zutreffende Wertermittlung für den Grund und Boden, sonst ist keine hinreichende Bodenmobilität zu erreichen. Und mit diesem Hinweis auf eine weitere komplexe Grundlage dieser Thematik ist der Einblick zu schließen.

\section{Literatur}

1. Weiß, E.; Zur Entwicklung des ländlichen Bodenrechts bei der Vereinigung Deutschlands. Vermessungswesen und Raumordnung (VR), 1992, Heft 1, S. 1-15.

2. Weiß, E. Verfassungsvorschriften der EG-Staaten zur Eigentumsgewährleistung. Zeitscrift des Bundes der öffentlich bestellten Vermessungsingenieure, (BDVIFORUM), 1992 Heft 3, S. 282-289.

3. Seele, W. Zur bodenpolitischen Bedeutung der Landumlegung. Vermessungswesen und Raumordnung, (VR), Heft 7, 1982, S. 353 ff.

4. Schmidt-Aßmann, E.; Gestalt und Wandelbarkeit der städtebaulichen Umlegung im Lichte des Art. 14 GG. StaatWirtschaft-Steuern, Festschrift für K. H. Friauf zum 65. Geburtstag, 1997.

5. Weiß, E.; Die städtebauliche Unternehmensflurbereinigung im Lichte der Fachliteratur; Vermessungswesen und Raumordnung, (VR), Heft 6/7, 1999, S. 1 ff..

6. Weiß, E.; Möglichkeiten der Unternehmensflurbereinigung zur Förderung von Fachplanungen. Zeitschrift für Vermessungswesen (ZfV), 1991, Heft 10, S. 420-441.

7. Weiß, E.; Die allgemeine Bodenfrage - eine immer aktuelle Aufgabenstellung unserer gesellschafts-politischen Entwicklungsmöglichkeiten. Vermessungswesen und Raumordnung (VR), Heft 7, 1998, S. 321-335.

\section{ŽEMĖS TVARKYMAS URBANIZUOTOSE VOKIETIJOS FEDERACINĖS RESPUBLIKOS TERITORIJOSE}

\section{E. Weiß}

\section{S a n tra u k}

Straipsnyje apžvelgiami svarbiausi Vokietijos Federacinès Respublikos žemès nuosavybès aspektai. Nagrinėjami žemės nuosavybès ístatymai ir Konstitucijos nuostatai. Aprašoma valstybinio žemès tvarkymo bei jos naudojimo planavimo VFR sistemos esmè ir pagrindai. Pristatomi svarbiausi viešieji teisiniai žemès naudojimo pertvarkymo būdai iškilus naujiems miestų pletros ir statybos, žemdirbystès bei gyvenviečių gerinimo, taip pat valstybès ir verslo poreikiams. Atsižvelgiama $\mathfrak{i}$ asmeninius ir kitus interesus, keičiant sklypus bei jų ribas iki atlygintino žemès nusavinimo.

Ivairiapusis žemès naudojimo planavimas VFR padeda išspręsti urbanizuotose teritorijose, ypač priemiesčiuose, iškylančias painias prieštaras ir išvengti planavimo bei investicijų klaidų. Pabrež̌iama, kad efektyvus žemės tvarkymo priemonių taikymas yra susijęs su teisingu žemès įkainavimu. 